\title{
Heilungschancen gut, Verträglichkeit verbesserungswürdig
}

\begin{abstract}
Die Chancen, mit einem Hodgkin-Lymphom geheilt zu werden, bewegen sich mittlerweile um die $90 \%$ und sogar darüber. Zu diesem Erfolg haben die Studien der GHSG (Deutsche Hodgkin Studiengruppe) entscheidend beigetragen. Der Schwerpunkt der Arbeit liegt jetzt vor allem auf besserer Verträglichkeit der Therapien und weniger Spätfolgen.
\end{abstract}

- Die Inzidenz der Hodgkin-Lymphome (HL) bewegt sich mit 3/100000/ Jahr im Mittelfeld der hämatologischen Neoplasien. Klinisch präsentieren sich die meist jüngeren Patienten mit zervikalen gummiartig geschwollenen Lymphknoten oder auch mit einem Mediastinaltumor. Die Symptomatik ähnelt der beim Non-Hodgkin-Lymphom (NHL) mit Nachtschweiß, Gewichtsverlust, gelegentlich auch generalisiertem Juckreiz, berichtete Dr. med. Bastian von Tresckow, Köln.

\section{Therapieschemata}

In der Chemotherapie kommen zwei Schemata zum Einsatz: ABVD (Adriamycin, Bleomycin, Vinblastin, Dacarbazin) und BEACOPP (Bleomycin, Etoposid, Adriamycin, Cyclophosphamid, Vincristin, Procarbacin, Prednison). Mit der Involved Field Strahlentherapie (IFRT) versucht man, die Bestrahlungsfelder möglichst klein und Nebenwir-

Die Therapie orientiert sich am Stadium der Erkrankung (Tab. 1) in Komkungen damit niedrig zu halten.

\section{Tabelle 1}

\section{Stadieneinteilung der Hodgkin-Lymphome}

Stadium I Befall einer einzigen Lymphknotenregion (IN) oder eines einzigen lokalisierten extranodalen Herdes (IE)

Stadium II Befall von zwei oder mehr Lymphknotenregionen auf einer Seite des Zwerchfells (IIN) oder lokalisierte extranodale Herde und Befall einer oder mehrerer Lymphknotenregionen auf einer Seite des Zwerchfells (IIE)

Stadium III Befall von zwei oder mehr Lymphknotenregionen auf beiden Seiten des Zwerchfells (IIIN) oder lokalisierte extranodale Herde auf beiden Seiten des Zwerchfells (IIIE)

Stadium IV Verbreiteter (disseminierter) Befall eines oder mehrerer extralymphatischer Organe mit oder ohne Befall von Lymphknoten

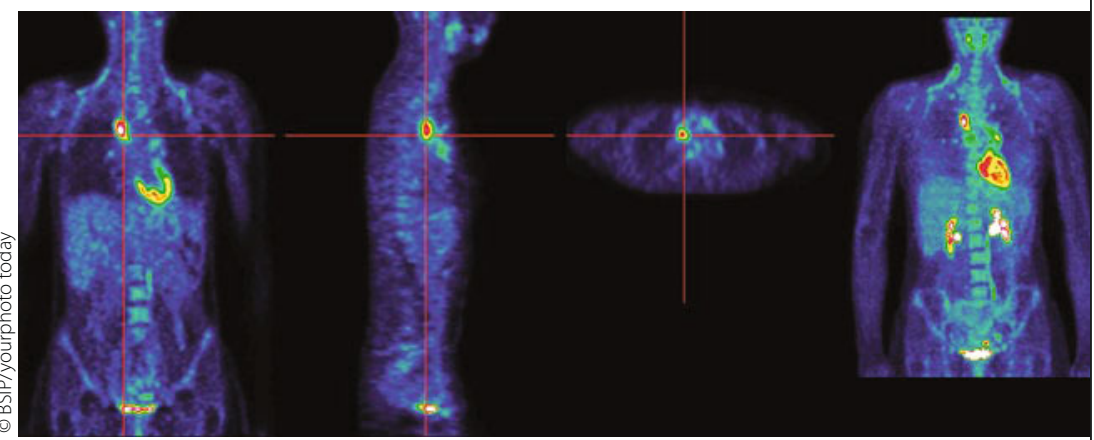

Hodgkin-Lymphom im Stadium III. bination mit vier Risikofaktoren $(\geq 3$ befallene Lymphknotenareale, erhöhte Blutsenkungsgeschwindigkeit, großer Mediastinaltumor und Extranodal-Befall). Für die frühen Stadien ist zweimal ABVD plus 20 Gray IFRT Therapie der Wahl, im mittleren Stadium zweimal BEACOPP eskaliert plus zweimal ABVD gefolgt von 30 Gray IFRT. Bei fortgeschrittenem HL gilt achtmal BEACOPP als Standard.

In der HD-15-Studie waren Patienten in fortgeschrittenen Stadien eingeschlossen. Mit der Absicht, die Verträglichkeit der Therapie zu verbessern, wurde ein Konzept gewählt, bei dem mit BEACOPP vorbehandelte Patienten nur dann eine Strahlentherapie mit 30 Gray erhielten, wenn sich beim Restaging und nach PET-Scan aktive Residuen $\geq 2,5$ $\mathrm{cm}$ fanden. „Es sieht so aus, dass man in Zukunft die Bestrahlung auf Patienten mit PET-positiven Resten beschränken kann“, erklärte von Tresckow.

\section{Gefürchtete Spätschäden}

Akuttoxizitäten mit der Folge von Infektionen, Fatigue, Übelkeit/Erbrechen sind meist Folge einer ungezielten Chemotherapie. Beim HL sind angesichts der meist jungen Patienten und des guten Langzeitüberlebens besonders die Spättoxizitäten bedeutsam. Hier spielen Sekundärneoplasien, v. a. die akute myeloische Leukämie, das NHL sowie solide Tumoren eine Rolle. Hinzu kommen Organschäden an Lunge, Herz, Schilddrüse und die Polyneuropathie.

Gerade beim HL ist es deshalb wichtig, stadienadaptiert $\mathrm{zu}$ therapieren und die Primärtherapie auf das Maß zu beschränken, das notwendig ist, um möglichst hohe Heilungsraten bei möglichst wenig Schädigung zu erzielen.

\section{MARTIN BISCHOFF}

- Symposium „Maligne Lymphome“ anlässlich der DGIM-Tagung am 1.5.2011 in Wiesbaden 